\title{
A probabilistic approach to modeling collisions with thin barriers
}

\author{
Sergey Pashkov* \\ Research Institute of Applied Mathematics and Mechanics, Tomsk State University, 36, Lenin av., \\ Tomsk, 634050 Russia.
}

\begin{abstract}
In many fracture problems, fragmentation is essentially a probabilistic process, which is determined by the stochastic nature of the distribution of inhomogeneities of the internal structure of material. The probabilistic approach is described, which allows us to model structural heterogeneities of the material in a simple form, practically without any complication of the model and additional experiments. Using experimental data and numerical simulation results, it is shown that the introduction of only one additional parameter (dispersion of the strength properties distribution) into the material model makes it possible to give a probabilistic character to the crack formation process at any scale level, which corresponds to theoretical concepts and experimental data. Distribution of materials strength characteristics (according to the selected distribution law) in the cells of the computational domain is used for initial heterogeneities and materials structure defects modeling. It is shown that the number and size of the "petals" at the penetration of thin barriers depend of the speed of the projectile and the strength characteristics of the barrier.
\end{abstract}

\section{Introduction}

In numerical simulation of dynamic interaction of deformable solids in many cases, it is necessary not only to assess the risk of damage to the body but also to reliably predict the nature of their destruction, size and shape of the resulting fragments. However, for most of these problems fragmentation is essentially a probabilistic process, which is determined by the stochastic nature of the distribution of inhomogeneities of the internal structure of the material [1]. This is especially evident for axisymmetric problems, for example, destruction of axisymmetric shells or cylindrical containers under explosive loading [2-4]. Despite the symmetry of the loading conditions, in the process of interaction there is a localization of deformations on the inhomogeneities of the structure of the material. Then stress relaxation zone is formed near the growing cracks, which depends on the strain rate and determines the characteristic size of the fragment. In the interaction of projectiles with barriers deformation localization also significantly affects the nature of the destruction and can lead to appearance of radial cracks (for brittle materials) or the formation of 'petals' on the backside of a relatively thin plastic barrier.

*Corresponding author: $\underline{\mathrm{s} 300 @ n g s . r u}$ 
For a long time, the probabilistic approach to solving such problems was limited to the analytical dependences of the fragmentation spectrum parameters on the strain rate determined by the Mott model and its variants proposed by Grady, Gilvarry and other researchers [1]. A large number of researchers in the modeling of fracture processes [3, 5-7] rely to some extent on the approach of Mott, which, in fact, is the evolution of a simplified one-dimensional model problem, which makes it insufficiently justified and difficult to apply in practical terms to determine fragmentation spectrum or fragments velocities of real structures destroyed by an explosion. Currently, the development of computer technology makes the most promising approach that allows for numerical simulation of destruction to take into account the heterogeneity of the body's internal structure by distributing the physical and mechanical characteristics responsible for strength [2-4, 6, 7]. Moreover, the regular ability to specify the distribution of structural inhomogeneities is already included in some popular software designed to calculate the destruction [7].

The probabilistic approach described in this article allows modeling structural heterogeneities of the material in fracture problems, thereby increasing accuracy, removing the limitations of the classical approach and solving problems in the most realistic formulation. This technique allows us to simulate the effect of initial inhomogeneities and structural defects on the nature of the dynamic destruction of solids in a simple form, almost without complicating the material model.

\section{A probabilistic approach}

\subsection{Modeling of structural inhomogeneities}

Taking into account the real internal structure of materials in numerical modeling cannot be a solution to the problem of the probabilistic nature of the destruction, since in most cases, this structure is unknown and its definition is a separate, very time-consuming task. Despite the fact that the direction of the study and modeling of the bodies internal structure is quite popular (the so-called multi-level approach), it is used mainly in solving problems of determining the effective (average) characteristics of materials with complex structure, at best for samples commensurate with the representative volume. For problems modeling the destruction of real objects, such an approach is practically inapplicable, since it requires rigorous research of the internal structure, and the creation of a workable, complex model for its numerical simulation, and an excessive level of detail of the grid (which slows down the calculation and imposes excessive demands on computing resources).

Moreover, the information about the real internal structure does not guarantee the probabilistic nature of the fracture, since the distribution of inhomogeneities is stochastic even in structured materials such as composites, and the internal structure of the real body has inhomogeneities at different levels (micro, meso, and macro).

The main obstacle for the probabilistic character of crack formation in the classical modeling approach is that there are no inhomogeneities in the structure of the material, which in a real situation provide the formation of microcracks and local stress relaxation in the adjacent volume at earlier stages of deformation. Therefore, in order to make the process probabilistic and close to reality, the distribution of strength characteristics in the volume of the sample should provide a certain spread of the limiting states of the material, at which the localization of deformations or the formation of microcracks begins. In this case, information about the real internal structure becomes redundant and it becomes possible to use effective values of strength characteristics even for composites in numerical modeling. 
In numerical simulation of fragmentation problems, with the grid-based methods or the meshless methods (for example, the SPH method [8]) using, the material is represented by discrete parameters bound to the nodes and cells of the computational domain. Therefore, for modeling of structure inhomogeneities it is necessary to introduce certain perturbations in the distribution of those parameters of the material model that are responsible for strength characteristics and determine the initiation and development of destruction. The described approach proposes to distribute the yield strength and the limit value of the damage parameter, which is used as a failure criterion. If the model of accumulation and growth of damage does not imply an explicit relationship between the selected parameters, their distributions are considered independent, since they depend on different defects in the structure (for example, defects on the grain boundary can prevent plastic deformations, but at the same time lead to earlier formation of microcracks).

The choice of the distribution law (normal, exponential, Weibull distribution and other multi parameter distributions) for the described probabilistic approach may cause a discussion. However, numerical experiments with the destruction of thick-walled cylindrical shells [2] have shown that the dispersion of the initial distribution of strength properties has a stronger influence on the formation of the fragmentation spectrum than its shape. Therefore, it reduces the requirements to the choice of the distribution law and allows for the analytical and numerical calculations with the same success to use almost any unimodal distribution. This is consistent with the conclusions [4] that the results of numerical modeling are not very sensitive to the choice of the distribution law. The determining influence of the distribution dispersion is consistent with the theoretical concepts - not all inhomogeneities and defects of the structure can be realized as fractures, but only those whose deviation from the average value exceeds a certain value.

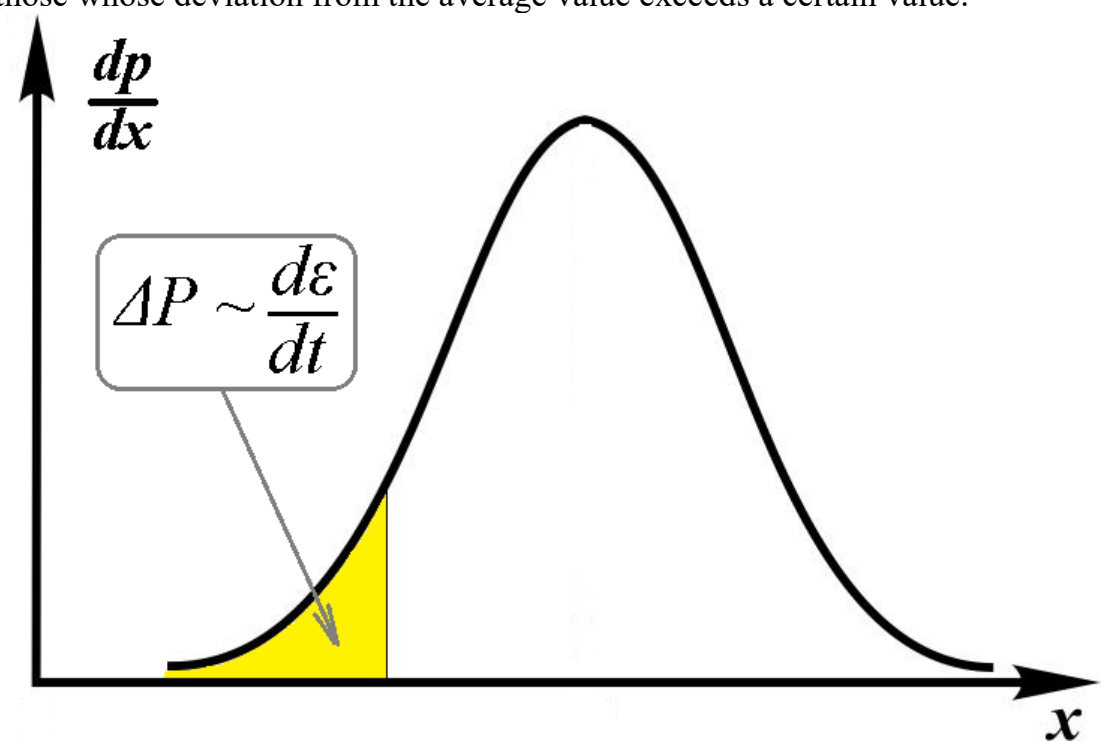

Fig. 1. The part of the distribution that determines the size of the fragments is bounded and depends on the strain rate.

In other words, the formation of the fragmentation spectrum for a particular problem is determined by a limited part (Fig. 1) of the limit values distribution function. The width of this area depends on the strain rate and is determined on the basis of the condition of propagation of the waves of unloading (from the first microcracks) on the entire volume of the destroyed sample. In the future, localization of deformations occurs, growth and fusion 
of cracks and the type of distribution function on the remaining range practically do not affect the large fragments of the fragmentation spectrum.

At present, the use of two-parameter Weibull distribution arising from the power probability function of distribution ('catastrophe function') in Mott theory is considered to be generally accepted for specifying the initial inhomogeneities in numerical modeling. Despite this, many researchers explicitly or implicitly recognize the lack of validity of this approach and the need to adjust the distribution parameters to match the experiment better. In our opinion, since we are discussing inhomogeneities at the micro level, a large number of relatively independent parameters affects their distribution. That, in fact, is the definition of the normal distribution law in probability theory. Therefore, it is proposed to use the normal (Gaussian) distribution as a sufficiently reasonable theoretically and convenient for modeling in contrast to the works [3, 5-7], which use the Weibull distribution.

The variants of the Weibull distribution used to describe the limit values distribution are usually very close to the normal law, but the use of two parameters that do not have an explicit physical meaning can lead to potential errors. In addition, random generators that generate a value obeying the Weibull distribution are not particularly common and this may deter many researchers from applying the probabilistic approach. The use of normal distribution, random generators for which are widespread for any programming language, greatly simplifies the problem.

\subsection{Distribution of initial inhomogeneities}

The spread of some value is described by the normal distribution (1) in the case when a large number of random independent parameters affects this value. Most locally distributed random variables in nature satisfy this requirement and, as a rule, can be considered as normally distributed with a sufficient accuracy in analytical and numerical calculations.

$$
\frac{d p}{d x}=\frac{1}{\sigma \sqrt{2 \pi}} e^{\frac{-\left(x-x_{0}\right)^{2}}{2 \sigma^{2}}}
$$

$\mathrm{x} 0$ - mathematical expectation;

$\sigma$ - standard deviation;

$\sigma^{2}-$ dispersion.

Since the normal distribution is defined on $[-\infty ; \infty]$, in practical applications it is required to limit the interval by eliminating areas where the probability is close to zero. Normal distribution makes it convenient to limit the interval, for example, according to the $3 \sigma$-rule.

\section{Modeling of dynamic fracture}

\section{1 'Petaling' of thin barriers}

We consider the proposed probabilistic approach in more detail with examples of modeling high velocity penetration through thin barriers. The penetration of thin barriers made of relatively plastic materials by the projectiles is usually accompanied by 'petaling'despite the initial symmetry of the task, the edges of the hole are torn, bent and have the form of separate 'petals'. The number of 'petals' generally depends on the impact velocity and strength characteristics of the material. Despite the uniformity and symmetry of the loading, in reality, localization of deformations on the inhomogeneities of the structure begins quite quickly. Then, near the growing cracks, a relaxed zone is formed, depending on the deformation rate and determining the characteristic size of the 'petal'. 
Using the software package PS300, developed at the Institute of Applied Mathematics and Mechanics of Tomsk State University, in the Lagrangian formulation with a tetrahedral grid, a numerical simulation of the problem of penetration of a thin metal plate by a projectile with a sharpened nose was carried out. The plate is $26.4 \mathrm{~cm}$ in diameter and 2.3 $\mathrm{mm}$ thick and is rigidly fixed at the edges. A projectile with a diameter of $6.6 \mathrm{~cm}$ was considered to be rigid, which, however, does not matter, as the barrier is too thin and a massive projectile almost was not deformed. The fracture criterion based on maximum deformations was used. When performing the fracture criterion at the node, a new contact surface was created using the method of grid splitting in nodes [9].

Figure 2 presents the results of numerical simulation of the problem of penetration of a projectile with sharpened nose through a thin plate. It is clearly seen how the change in the parameters of the experiment changes the number of 'petals'. For copper plate, it is accepted: yield strength $=200 \mathrm{MPa}$; elongation at break $=0.563$. For a steel plate, it is accepted: yield strength $=940 \mathrm{MPa}$; elongation at break $=0.2$.

Within the framework of the described probabilistic approach, each cell has an independent value of the ultimate plastic deformation and yield strength. The parameters responsible for strength were distributed over the cells of the computational domain by a modified random generator, which produces a random value distributed according to the normal law (1) with the expectation $\mathrm{x}_{0}$ corresponding to the table value and dispersion corresponding to the $10 \%$ interval [2], defined by $3 \sigma$-rule.

Steel plate (Fig. 2a), due to the lower plasticity, has less time to localize the damage at the initial defects of the structure and less time to relax in the event of a crack, so at the same speed of the projectile $300 \mathrm{~m} / \mathrm{s}$ has a greater number of 'petals' than the copper plate (Fig. 2b). For the copper plate, the calculations were made at different projectile velocities. If the speed of the projectile is reduced to $150 \mathrm{~m} / \mathrm{s}$ (Fig. 2c), the time for localization of damage and stress relaxing in the event of a crack, the unloading zone (and, accordingly, the size of the fragment) increases, so the number of petals reduced to four.
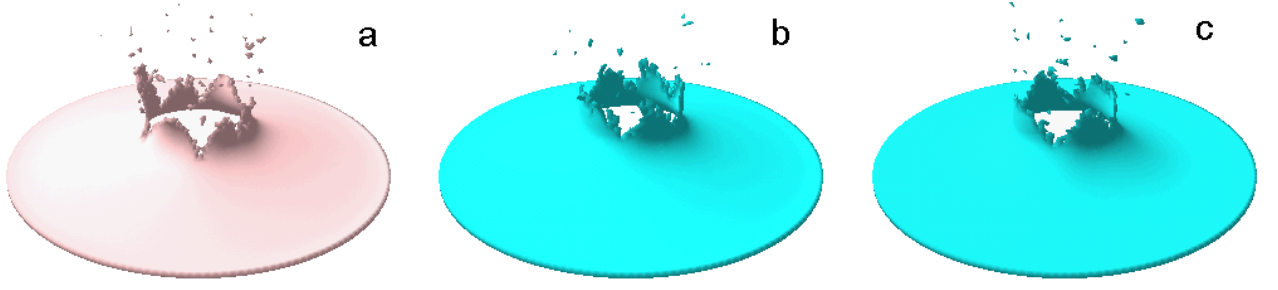

Fig. 2. Penetration of a thin metal plate by a projectile with a sharpened nose: a) steel plate, the velocity of the projectile $300 \mathrm{~m} / \mathrm{s}, 6$ 'petals'; b) copper plate, velocity of the projectile $300 \mathrm{~m} / \mathrm{s}, 5$ "petals"; c) copper plate, velocity of the projectile $150 \mathrm{~m} / \mathrm{s}, 4$ 'petals'.

These results show that the proposed probabilistic approach allows even in a geometrically axisymmetric problem to describe the localization of deformations on the defects of the structure, provide the 'petaling' observed in experiments, and confirm the dependence of the number of 'petals' on the velocity of the projectile and the plastic properties of the barrier.

In order to verify the approach used, the experimental data were compared with the results of numerical simulations.

Figure $3 \mathrm{a}$ shows the result of an experiment on the penetration of a thin metal barrier conducted at the research Institute of Applied Mathematics and Mechanics of Tomsk State University with a light-gas gun [10]. The plate thickness of $1.7 \mathrm{~mm}$ is made of alloy $\mathrm{AMg} 6$, used in the protective shields of spacecraft. An aluminum spherical projectile with a diameter of $9 \mathrm{~mm}$, imitating a micrometeoroid, was accelerated to a speed of about 800 $\mathrm{m} / \mathrm{s}$. The experiment shows the characteristic ragged edges and a sufficiently large 
(compared to the size of the projectile) hole size, due to the flattening of the aluminium ball during the penetration.

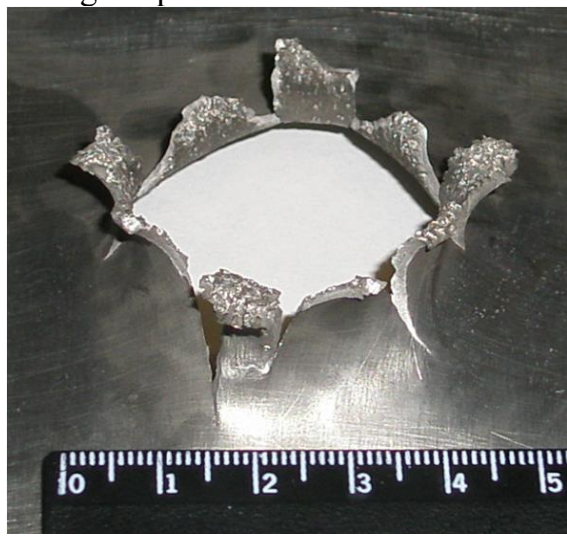

a

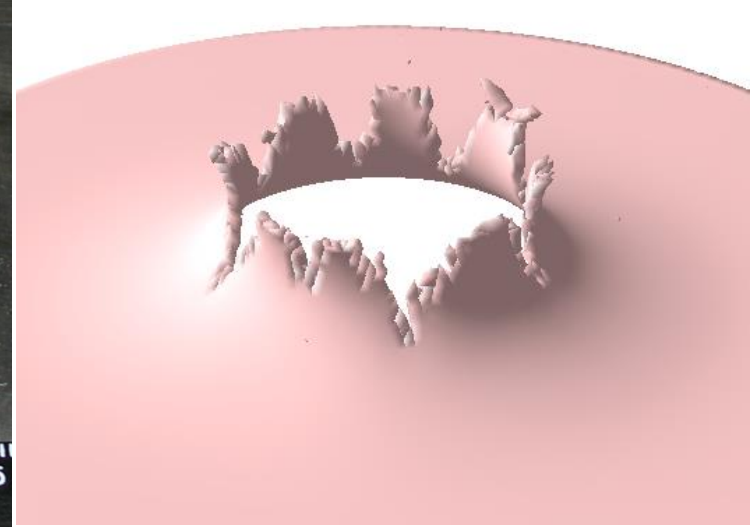

b

Fig. 3. Penetration of a plate $1.7 \mathrm{~mm}$ thick from the AMg6 alloy by an aluminium spherical striker with a diameter of $9 \mathrm{~mm}$, an impact velocity of about $800 \mathrm{~m} / \mathrm{s}$; a) experiment; b) numerical simulation.

In this calculation, the probabilistic approach in the form of the initial distribution of the yield strength and limit deformations according to the normal law was also applied. The use of the probabilistic approach for this problem showed a good agreement between the experimental data and the results of numerical simulation both in the number of 'petals' and in their shape.

\subsection{The influence of strength properties distribution's dispersion to the characteristic size of the fragment}

The described probabilistic approach can be successfully used not only for 'petaling' modeling, but also for other problems where the destruction is stochastic, when the fracture strongly depends on the deformation rate and the localization of damage on the structure defects and internal inhomogeneities. For example, explosive destruction of thick-walled shells [11-12]. Since the described approach involves introduction of an additional parameter (dispersion of strength properties) into the material model, it is interesting to study the effect of dispersion of strength properties on the characteristic size of the fragment and the fragmentation spectrum.

The calculations of the simulation of explosive fracture of shells show [2] that the increase in the dispersion of strength properties leads to an increase in the spectrum part of medium and large fragments and to a decrease in the maximum caused by small fraction fragments, therefore the average size of the fragment increases. The maximum size of the fragment also tends to increase, which leads to an increase in the number of fragments containing portions of both initial surfaces (internal and external). This is due to the fact that the number of large defects implemented in microcracks in the initial stages of destruction, increases with increasing dispersion, and stress relaxing from the newly formed surfaces reduces the risk of smaller defects.

At the same time, it should be understood that for some problems the increase in dispersion from a certain value ceases to significantly affect the fragmentation spectrum because at high strain rates the localization of damage begins to appear only at a certain number of microcracks. 


\subsection{The influence of grid step}

When considering this model, there may be a question of the validity of binding structure defects to the grid and assessing the impact of cell size on the resulting fragmentation spectrum because when changing the cell size, the spatial distribution of the initial inhomogeneities will change fundamentally. This point was analyzed in detail and the following conclusions were made. The selected grid step, in fact, determines the level of the structure modeling, at the same time at any level there are distributed real inhomogeneities, which act as triggers and initiate the formation of microcracks. At the atomic level, the inhomogeneities are dislocations and other defects of the crystal lattice. In larger scale analysis, grains and grain boundaries act as defects. In macro analysis, the inhomogeneities are already microcracks and other relatively large stress concentrators. Thus, the approach with the distribution of strength characteristics is applicable for any cell size, however, as in any other algorithm using grid approximation, the cell size should be sufficient to ensure the necessary accuracy (in this case - the accuracy of the definition of the fragmentation spectrum).

For most problems, the reduction of the grid step causes an increase in the number of fragments of the fine fraction but does not lead to a noticeable change in the fragmentation spectrum in the coordinates of 'mass by mass'. Since the main role of the initial inhomogeneities is the concentration of stresses in the formation of the first microcracks, and the size of the fragment, in fact, is determined by the deformation rate. Nevertheless, it is possible that in the problems of crushing brittle materials (very sensitive to the choice of the fracture model), a decrease in the grid step can lead to a change in the fragmentation spectrum and in the part of large fragments. General advice here is to specify the variance of the distribution for a given level of modeling of the structure using the compare to experiment.

\section{Conclusion}

Using experimental data and numerical simulation results, it is shown that introduction of only one additional parameter (dispersion of the strength properties distribution) into the material model makes it possible to give a probabilistic character to the cracking process at any scale level. The proposed probabilistic approach can be used at any step of the grid and at any level of multilevel modeling, providing the distribution of inhomogeneities of the characteristic size. This makes it possible to improve the accuracy of calculations and remove the limitations of the classical approach to numerical modeling of fracture problems, solving them in the most realistic formulation. Within the framework of this approach, only a stochastic factor is introduced, so no special experiments are required for preliminary study of the material structure. The approach can be applied to any material models and fracture criteria.

The Russian Science Foundation (RSF), project no. 16-19-10264, supported this work.

\section{References}

1. O.B. Naimark, S. V. Uvarov, M. M. Davydova, I. A. Bannikova, Physical Mesomechanics, 20, 90-101(2017)

2. S.V. Pashkov, Proc. of Tomsk state University, 302, 204-211 (Tomsk State University, Tomsk, 2018) 
3. D.E. Lambert, J. Weiderhold, J. Osborn, M.V. Hopson, J. of Pressure Vessel Technology, Transactions of the ASME, 134, 031209 (2012)

4. Q.B. Diep, J.F. Moxnes, G. Nevstad, Proc. 21th Int. Symp. on Ballistics, 752 (Adelaide, Australia, 2004)

5. P. Elek, S. Jaramaz, Theoretical and Applied Mechanics, 32, 113-130 (2005)

6. H.W. Meyer, R.M. Brannon, Int. J. of Impact Engineering, 42, 48-58 (2012)

7. J.G. Rasico, C.A. Newman, M.R. Jensen, Int. J. of Vehicle Performance, 4, 323-346 (2018)

8. R. O. Cherepanov, A. V. Gerasimov, AIP Conference Proceedings, 1783, 020024 (2016)

9. A.V. Gerasimov ed., High-speed impact. Modelling and experiment (Scientific and Technical Literature Publishing House, Tomsk, 2016)

10. A.V. Gerasimov, D.B. Dobritsa, S.V. Pashkov, Y.F. Khristenko, Cosmic Research, 54, 118-126 (2016)

11. A.V. Gerasimov, S.V. Pashkov, Khimicheskaya Fizika, 21, 34-36 (2002)

12. A.V. Gerasimov, S.V. Pashkov, Khimicheskaya Fizika, 24, 1-7 (2005) 